\title{
Graves' orbitopathy- When the going gets tough... the tough get going?
}

Authors: Alexandra Nila1 ${ }^{1}$, Cristina Serbanescu ${ }^{1}$, Minodora Betivoiu ${ }^{1}$, Sorina Martin ${ }^{1,2}$, , Simona Fica ${ }^{1,2}$

1. Elias Hospital, Endocrinology Department

2. Carol Davila University of Medicine and Pharmacy, Endocrinology Department

\section{INTRODUCTION}

Thyroid disorders are common within the elderly population and are particularly challenging to diagnose and treat due to the presence of a wide variety of comorbidities. Graves' ophtalmopathy remains a therapeutic predicament despite all progress made in understanding its pathogenesis, especially in the elderly population with severe presentation.

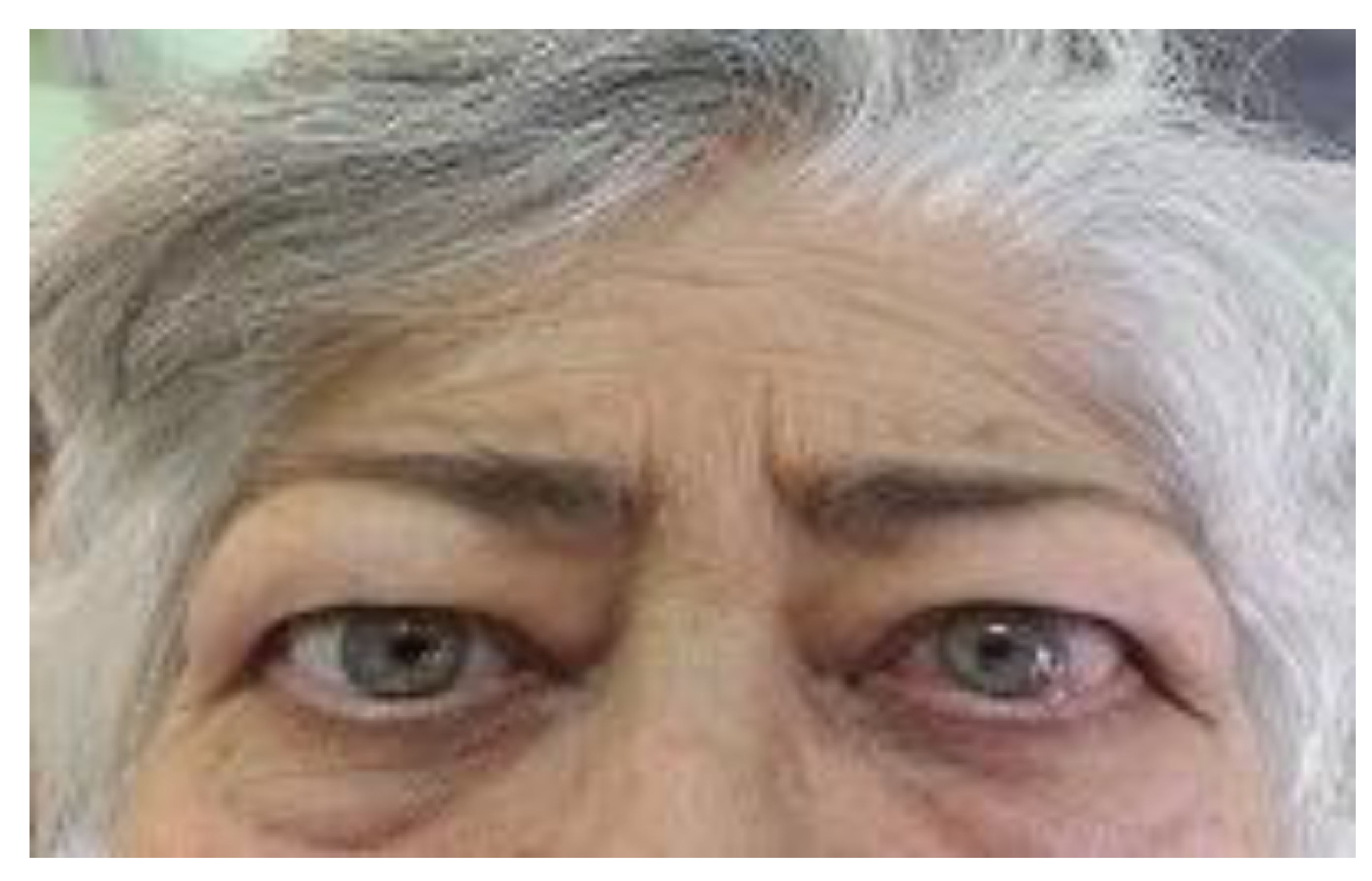

Figure 1- clinical presentation at admission

\section{CASE PRESENTATION}

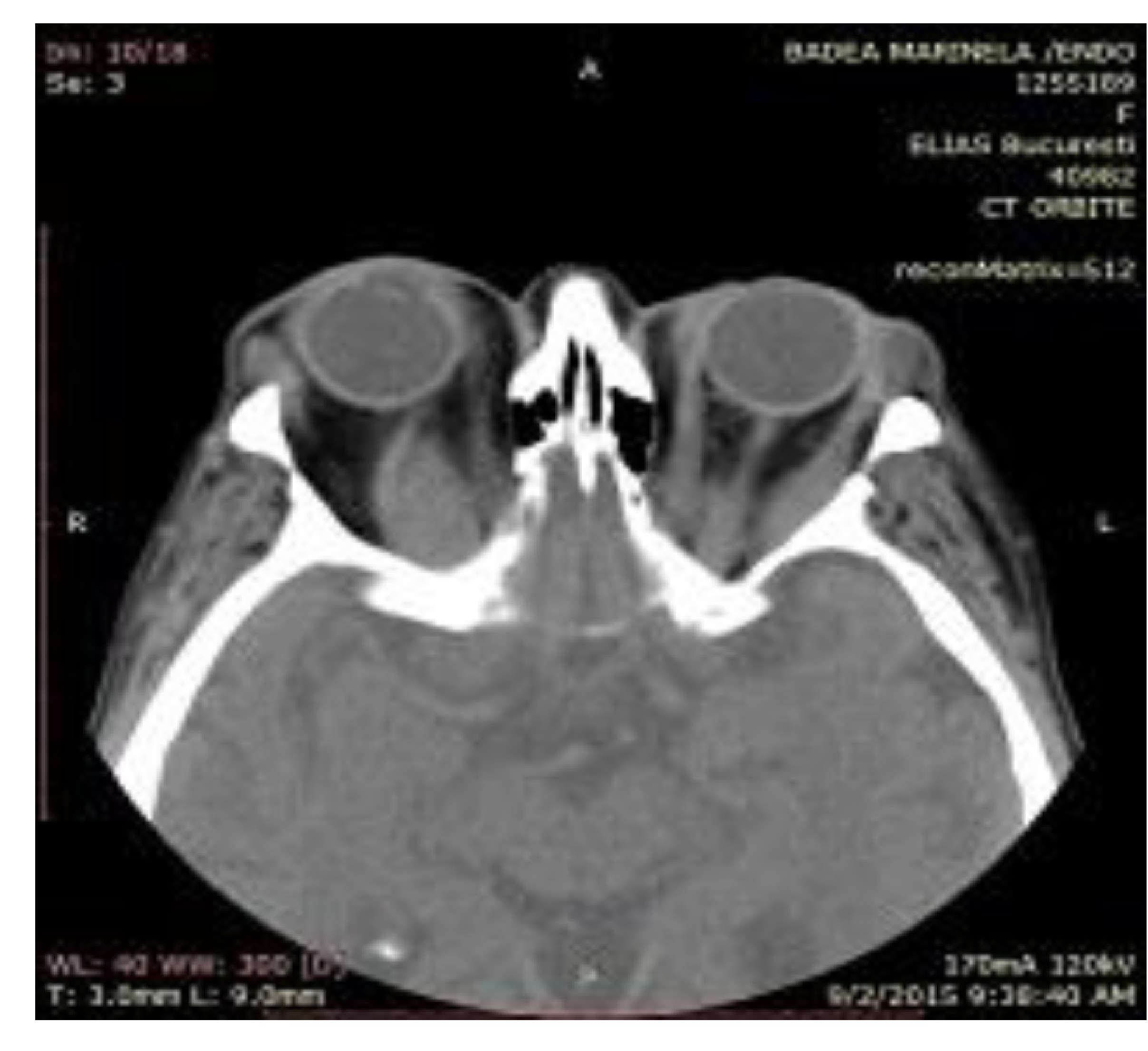

Figure 3- orbit CT scan
We present the case of a 71-yrs old female patient who was admitted in our clinic for diplopia, decreased visual acuity, visual field amputation, dysphonia and thyroid enlargement. She was diagnosed with Graves' disease 2 years prior her admission and had been on block and replace treatment ever since. Her medical history includes: class III NYHA heart failure, metallic mitral valve replacement, permanent atrial fibrillation, severe tricuspid regurgitation and severe secondary pulmonary hypertension. Clinical examination (Fig. 1) revealed bilateral exophthalmia, eyelid edema, conjunctival hyperemia, convergent strabismus and a large, compressive goiter. Lab tests revealed iatrogenic hypothyroidism (TSH=36.7 ulU/ml,FT4<0.30 ng/ml,TT3-=104.3 ng/dl).

The orbit CT scan (Fig. 2) and ophtalmological examination (Fig. 3) discovered bilateral hypertrophy of orbital rectus muscles, disthyroid optic neuropathy, paracentral scotoma, very low motility and corneal edema in both eyes. The cardiologic examination pointed out her high surgical risk considering the heart failure and severe pulmonary hypertension. The Methimazol dose was adjusted and over the next 4 months, under multidisciplinary surveillance, our patient received 3 courses of pulse therapy with low-dose methylprednisolone (375 mg over 2 courses and $250 \mathrm{mg} /$ one course, cumulative dose $1 \mathrm{~g}$ ), well tolerated, with slight improvement in visual field defects and visual acuity and amelioration of symptoms (Fig. 4). Considering the severe ophtalmopathy and the cardiac pathology, the patient continues treatment with Methimazol.
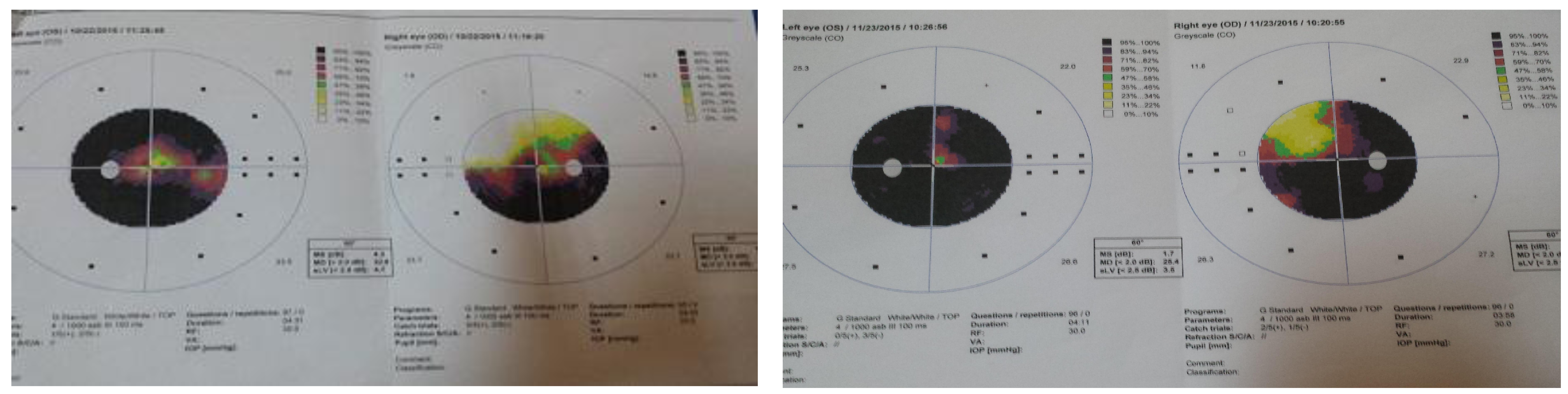

Figures 3 and 4: ophtalmological exam before and after methylprednisolone therapy

\section{CONCLUSIONS}

Treating Graves' disease/ orbitopathy can be challenging when having a severe presentation, especially in the geriatric population where associated pathologies, concomitant medication, treatment tolerance must be considered. 